\title{
Proceedings
}

\section{Quarkonia Production and Dissociation in a Langevin Approach ${ }^{\dagger}$}

\author{
Nadja Krenz *, Hendrik van Hees and Carsten Greiner \\ Institut für Theoretische Physik, Goethe-Universität Frankfurt, Max-von-Laue-Str. 1, D-60438 Frankfurt am \\ Main, Germany; hees@th.physik.uni-frankfurt.de (H.v.H.); Carsten.Greiner@th.physik.uni-frankfurt.de (C.G.) \\ * Correspondence: krenz@th.physik.uni-frankfurt.de \\ + Presented at Hot Quarks 2018-Workshop for Young Scientists on the Physics of Ultrarelativistic \\ Nucleus-Nucleus Collisions, Texel, The Netherlands, 7-14 September 2018.
}

Published: 17 April 2019

check for updates

\begin{abstract}
We aim to describe the process of dissociation and recombination of quarkonia in the quark-gluon plasma. Therefore we developed a model which allows to observe the time evolution of a system with various numbers of charm-anticharm-quark pairs at different temperatures. The motion of the heavy quarks is realized within a Langevin approach. We use a simplified version of a formalism developed by Blaizot et al. in which an Abelian plasma is considered where the heavy quarks interact over a Coulomb like potential. We have demonstrated, that the system reaches the expected thermal distribution in the equilibrium limit.
\end{abstract}

Keywords: heavy quarks; Langevin equation; quarkonia; charmonium; transport theory; recombination

\section{Introduction}

Heavy quarks are an important tool for the investigation of the quark-gluon plasma (QGP). They are primarily produced in the primordial hard collisions of the heavy ion collision, and their number is conserved until the hadronic freezout. Therefore heavy quarks carry information about the whole evolution of the QGP. Especially the surviving probability of heavy-quark bound states such as $\mathrm{J} / \psi$ or $\mathrm{Y}$ can give an insight to the medium properties.

The potential between two heavy quarks is screened by the surrounding medium. As suggested long ago, the suppression of $\mathrm{J} / \psi$ could be an evidence for the formation of the deconfined state [1]. Higher temperatures should lead to larger screening effects with a full suppression of $\mathrm{J} / \psi$ at very high beam energies. The predicted suppression was found at the SPS at CERN [2] but measurements at RHIC at higher beam energies did not show an increase of the suppression [3]. To explain the results the process of recombination of $\mathrm{J} / \psi$ inside the medium has been suggested. The theoretical investigation of recombination processes is therefore necessary to predict the number of $\mathrm{J} / \psi$-mesons measured in the experiments.

The comparatively large masses of heavy quarks makes their motion accessible by Langevin dynamics [4]. The forces that act on the charm quarks by using the Langevin equation are a drag force and random momentum kicks due to collisions with the medium particles. To enable the formation of bound states we add a potential between the heavy quarks.

\section{Formalism}

For the realization of the heavy-quark motion we adopt the formalism by Blaizot et al. [5]. In this description the heavy-quark interaction is reduced to an Abelian model, which means that confinement and color effects are neglected. The Langevin update rules for the coordinate $x$ and the momentum $p$ of a heavy quark with mass $M$ read 


$$
\frac{\mathrm{d} r}{\mathrm{~d} t}=\frac{1}{2 M} \boldsymbol{p} \quad \text { and } \quad \frac{\mathrm{d} \boldsymbol{p}}{\mathrm{d} t}=-\gamma \boldsymbol{p}+\boldsymbol{F}(\boldsymbol{r}-\overline{\boldsymbol{r}})+\sqrt{2 M T \gamma \Delta t} \rho,
$$

where $\gamma$ is the friction coefficient due to the interaction with the medium, $\boldsymbol{F}(\boldsymbol{r}-\overline{\boldsymbol{r}})$ is the force resulting from the heavy-quark potential, $T$ is the temperature of the medium, and $\rho$ are Gaussian normal-distributed random numbers. For the pertinent diffusion coefficient the usual Einstein dissipation-fluctuation relation has been employed. The quark-anti-quark potential is given by a screened Coulomb potential with a cut-off for large momenta at small distances. Following [5] the cut-off is taken to be $\Lambda=4 \mathrm{GeV}$. The potential for different temperatures is displayed in Figure 1 .

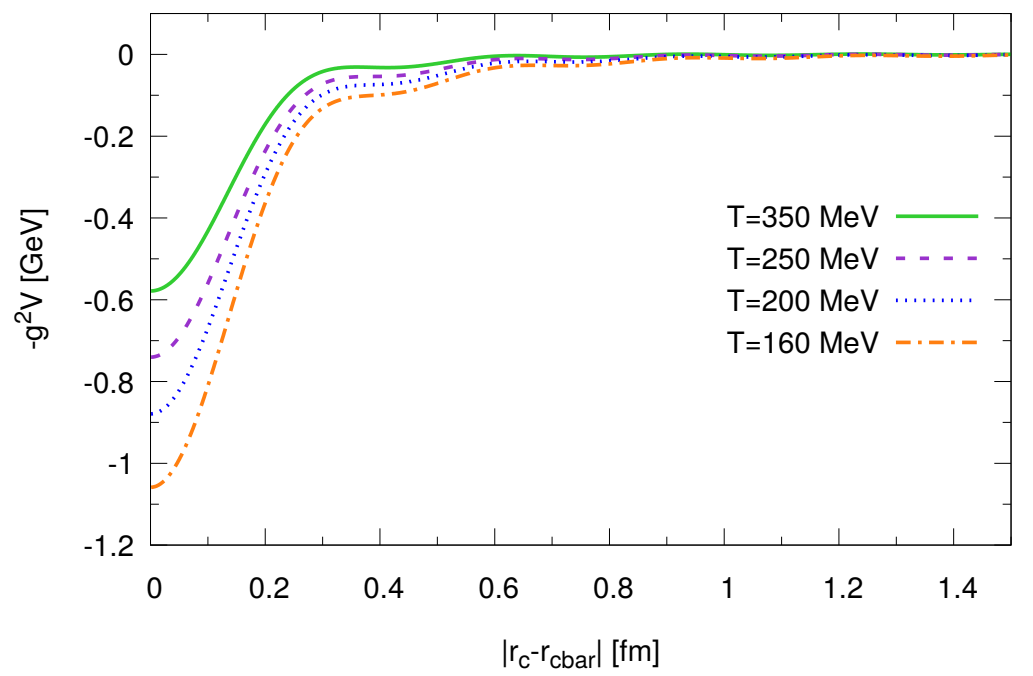

Figure 1. The charm-anti-charm-quark pair potential for different temperatures.

The drag force in [5] contains a dependence on the distance between the heavy quarks. For simplicity we neglect this dependence in our simulation and use a constant drag coefficient. In a numerical calculation a cut-off is also necessary for the friction. With the same cut-off as for the potential the drag coefficient is given by

$$
\gamma=\frac{m_{\mathrm{D}}^{2} g^{2}}{24 \pi M}\left[\ln \left(1+\frac{\Lambda^{2}}{m_{\mathrm{D}}^{2}}\right)-\frac{\frac{\Lambda^{2}}{m_{\mathrm{D}}^{2}}}{\frac{\Lambda^{2}}{m_{\mathrm{D}}^{2}}+1}\right]
$$

where $m_{\mathrm{D}}$ is the Debye screening mass, defined as $m_{\mathrm{D}}^{2}=\frac{4}{3} g^{2} T^{2}$, which is the perturbative expression for a two-flavor quark-gluon plasma. The gauge coupling $g$ is given by the relation [6]

$$
g^{2}=4 \pi \alpha_{s}=\frac{4 \pi \alpha_{s}\left(T_{C}\right)}{1+C \ln \left(\frac{T}{T_{C}}\right)}, \quad \text { with } \quad C=0.76, \quad T_{C}=160 \mathrm{MeV}, \quad \alpha_{s}\left(T_{C}\right)=0.5,
$$

where $T_{C}$ is the critical temperature. The value of $\gamma$ at different temperatures can be seen in Figure 2 . The charm-quark mass is set to $M=1.8 \mathrm{GeV}$. 


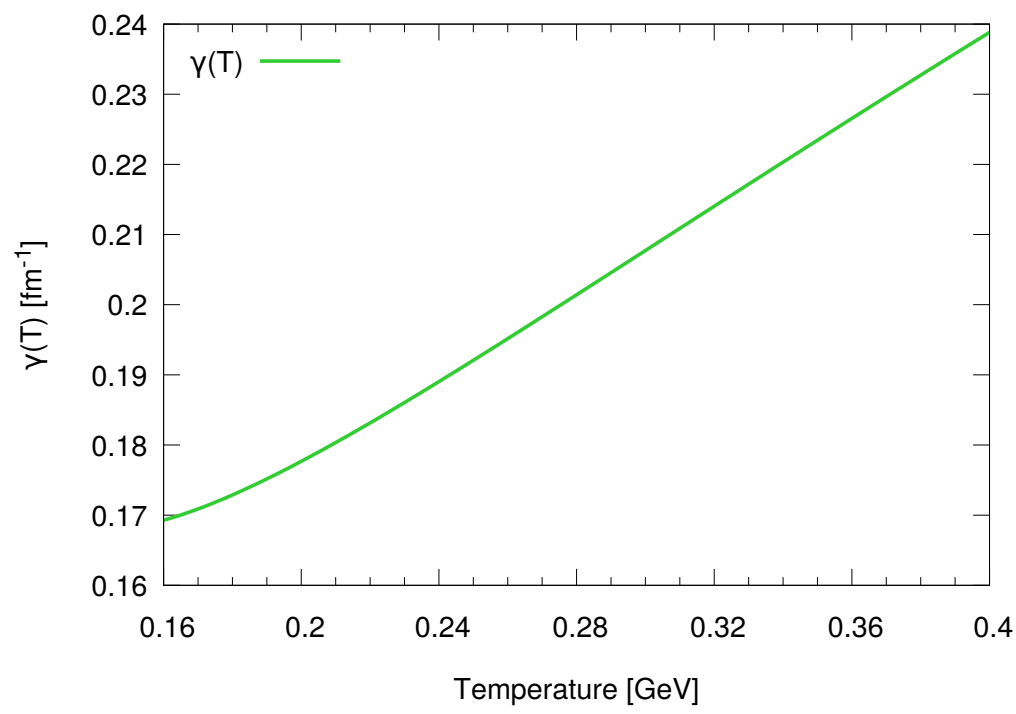

Figure 2. The friction coefficient for different temperatures.

\section{Results and Discussion}

First the simulation was tested with a single charm-anticharm pair in the medium. The following simulations are done inside a cubic box with side-length $8 \mathrm{fm}$. To define bound states we use the classical condition that two objects are bound if the energy of the pair is negative. We calculate the relative energy $E_{\text {rel }}$ of the pair, which means subtracting the center-of-mass energy from the total energy. After the system reaches equilibrium the distribution of the relative energy should be given by the classical density of states

$$
\frac{\mathrm{d} N}{\mathrm{~d} E_{\text {rel }}}=C \int_{\mathbb{R}^{3}} \mathrm{~d}^{3} r \int_{\mathbb{R}^{3}} \mathrm{~d}^{3} \boldsymbol{p}_{\text {rel }} \delta\left(E_{\text {rel }}-H_{\text {rel }}\right) \exp \left(-\frac{H_{\text {rel }}}{T}\right),
$$

where $H_{\text {rel }}$ is the Hamiltonian of the pair and $C$ is a normalization constant. The results are shown in Figure 3. For this plot both curves are normalized to one. We see that the numerical calculation perfectly fits to the analytic function.

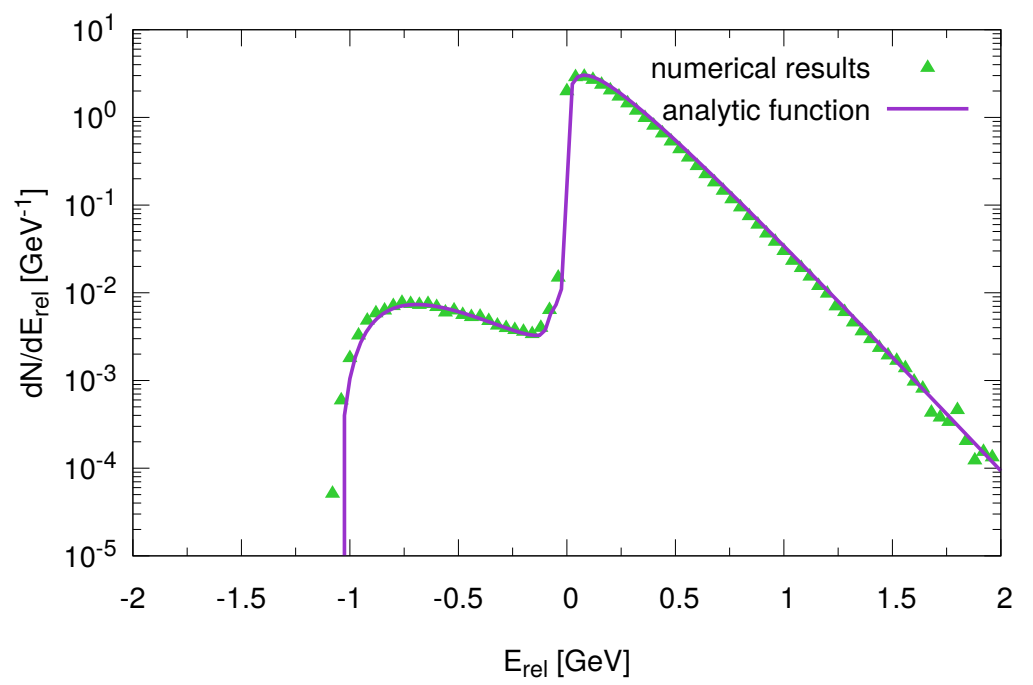

Figure 3. Pair-energy distribution at equilibrium. The simulation is done assuming a cubic box with boxsize $8 \mathrm{fm}$ at a temperature of $T=160 \mathrm{MeV}$.

We also found this agreement for different temperatures, as can be seen in Figure 4. For higher temperatures the number of bound states in equilibrium decreases due to stronger screening effects. 


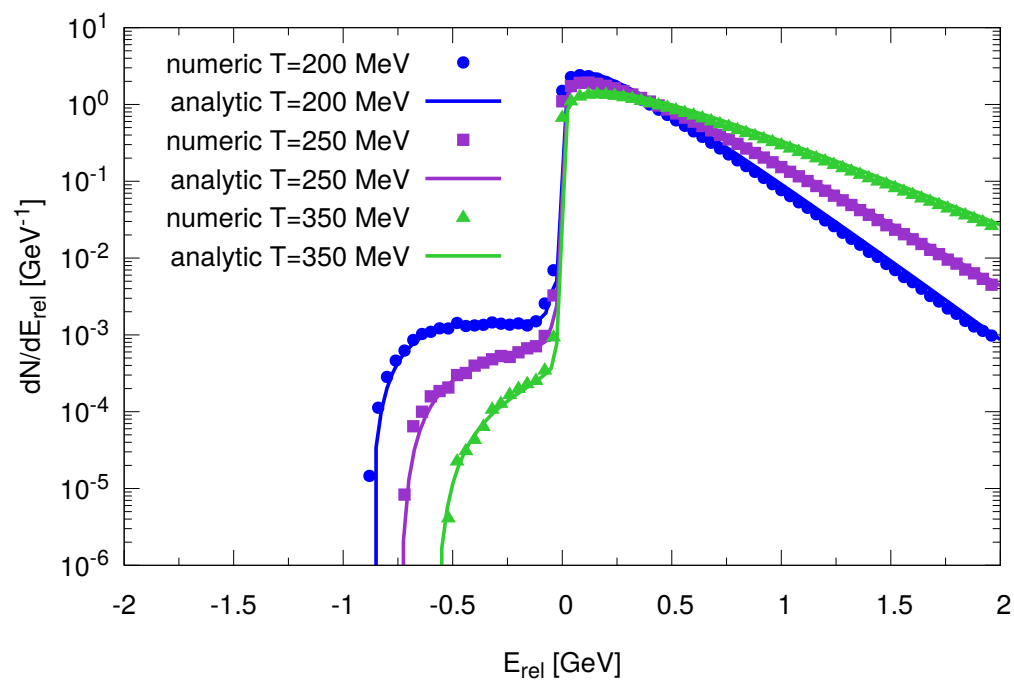

Figure 4. Pair-energy distribution for different temperatures. Due to stronger screening effects higher temperatures lead to a smaller yield of bound states.

We have also investigated the time evolution of the bound states at a temperature of $T=160 \mathrm{MeV}$ for two initial conditions.

In the first simulation the charm and anti-charm quarks are initially randomly placed inside the box. For the second simulation the heavy quarks are initially created as bound states with a pair energy of $-700 \mathrm{MeV}$, which approximately corresponds to the maximum of the peak on the left panel of Figure 3. The momenta are set back-to-back with a relative velocity that is taken from a Maxwell distribution with its center at the average value of typical relative velocities of charmonium $v_{0}^{2} \sim 0.3$ [7]. As shown in Figure 5, in both cases the fraction of bound states in the system equilibrates to the same value as expected. At this point the dissociation and recombination rates are equal and therefore the principle of detailed balance is fulfilled.

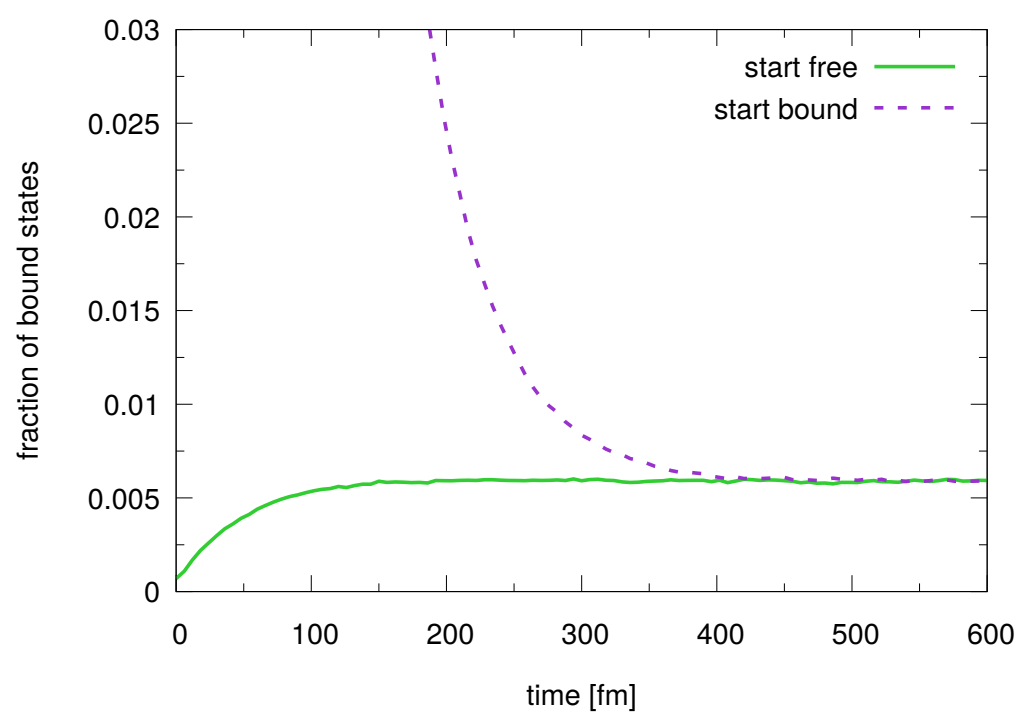

Figure 5. Time evolution of the fraction of bound states with the charm-anticharm pairs produced initially bound (violet dashed line) or placed randomly inside the box (green solid line). Both curves lead to the same equilibrium limit but the equilibration time is longer in case of initial bound states.

To see the influence of the medium's temperature on the equilibration time we calculated the time evolution at different temperatures. In this simulation we produced five charm-anticharm pairs, initially created as bound states. The results are displayed in Figure 6. 


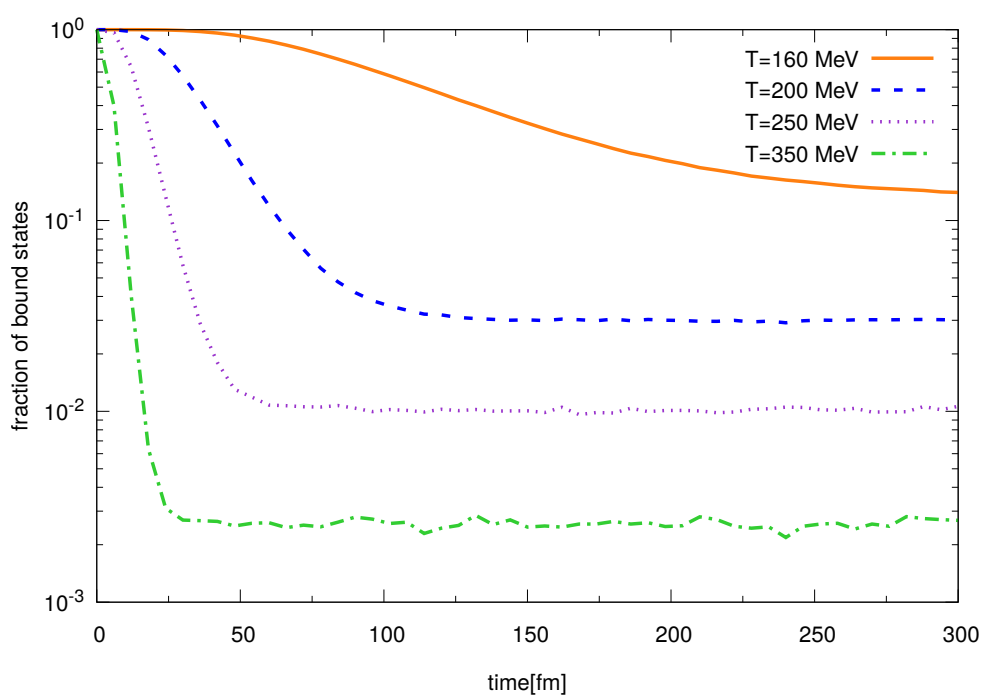

Figure 6. Time evolution of the fraction of bound states for different temperatures. Higher temperatures lead to a less amount of bound states and to a faster equilibration.

Larger screening effects at higher temperatures lead to a smaller fraction of bound states at equilibrium. Also the equilibration time decreases. This is expected, because the friction coefficient increases with temperature. The momentum and therefore also the energy transfer in the collisions with the light medium constituents is higher.

To check if our simulation is also in accordance with an equilibrated thermodynamic system, we calculated the particle number using the grand canonical partition function. Since the dissociation and recombination of a J/ $\psi$-meson is a process of the kind $\mathrm{J} / \psi \rightleftarrows c+\bar{c}$, the chemical potentials are connected by $\mu_{\mathrm{J} / \psi}=2 \mu_{c}$ which means for the fugacity $\lambda_{\mathrm{J} / \psi}=\lambda_{c}^{2}$. The number of $\mathrm{J} / \psi$ in the system can then be calculated by knowing the initial number of charm-anticharm-pairs. We compared the results from our simulation with those obtained by using the grand canonical partition function for three different box sizes in Table 1. We find that both values are of the same order of magnitude.

Table 1. Comparison between the fraction of bound states obtained with our simulation and that calculated by using the grand canonical partition function

\begin{tabular}{ccc}
\hline Volume & Grand Canonical Fraction of $\mathbf{J} / \boldsymbol{\psi}$ & Numerical Fraction of $\mathbf{J} / \boldsymbol{\psi}$ \\
\hline $8^{3} \mathrm{fm}^{3}$ & 0.0066 & 0.0059 \\
$10^{3} \mathrm{fm}^{3}$ & 0.0035 & 0.0029 \\
$12^{3} \mathrm{fm}^{3}$ & 0.002 & 0.0017 \\
\hline
\end{tabular}

We could show that our model passes all equilibrium tests. Various extensions are possible to improve the model. The long-time goal of this project is to obtain a full in-medium quantum Langevin treatment of quarkonia.

Funding: This work was supported by the DFG through the grant CRC-TR 211.

Conflicts of Interest: The authors declare no conflict of interest.

\section{References}

1. Matsui, T.; Satz, H. J/ $\psi$ Suppression by Quark-Gluon Plasma Formation. Phys. Lett. B 1986, 178, 416-422, doi:10.1016/0370-2693(86)91404-8.

2. Baglin, C.; Ramos, S.; Ferreira, R.; Drapier, O.; Guillaud, J.P.; Kluberg, L.; Racca, E.; Fredj, L.; Abreu, M.C.; Busson, P.; et al. Production de $J / \psi$ dans les collisions de proton, oxygène et soufre sur cibles lourdes, á 200 GeV/N. Can. J. Phys. 1989, 67, 1222-1227. doi:10.1139/p89-205 
3. Abelev, B. I. et al (STAR Collaboration) $J / \psi$ production at high transverse momenta in $p+p$ and $\mathrm{Cu}+\mathrm{Cu}$ collisions at $\sqrt{s_{N N}}=200 \mathrm{GeV}$. Phys. Rev. C 2009, 80, 041902, doi:10.1103/PhysRevC.80.041902.

4. Svetitsky, B. Diffusion of charmed quarks in the quark-gluon plasma. Phys. Rev. D 1988, 37, 2484-2491, doi:10.1103/PhysRevD.37.2484.

5. Blaizot, J.P.; De Boni, D.; Faccioli, P.; Garberoglio, G. Heavy quark bound states in a quark-gluon plasma: Dissociation and recombination. Nucl. Phys. A 2016, 946, 49-88, doi:10.1016/j.nuclphysa.2015.10.011.

6. Lettessier J and Rafelski J. Hadrons and Quark-Gluon Plasma, Cambridge University Press: Cambridge, New York, Melbourne, Madrid, Cape Town, 2004.

7. Bodwin, G.T.; Kang, D.; Kim, T.; Lee, J.; Yu, C. Relativistic Corrections to e+ e- $\longrightarrow$ J/psi + eta(c) in a Potential Model. AIP Conf. Proc. 2007, 892, 315-317, doi:10.1063/1.2714404.

(C) 2019 by the authors. Licensee MDPI, Basel, Switzerland. This article is an open access article distributed under the terms and conditions of the Creative Commons Attribution (CC BY) license (http:/ / creativecommons.org/licenses/by/4.0/). 